\title{
The metals-to-dust ratio to very low metallicities using GRB and QSO absorbers; extremely rapid dust formation
}

\author{
Tayyaba Zafar ${ }^{1}$ and Darach Watson ${ }^{2}$
}

\author{
1 Aix Marseille Université, CNRS, LAM (Laboratoire d'Astrophysique de Marseille) UMR 7326, 13388 Marseille, France \\ e-mail: tayyaba.zafar@oamp.fr \\ 2 Dark Cosmology Centre, Niels Bohr Institute, University of Copenhagen, Juliane Maries Vej 30, 2100 Copenhagen, Denmark
}

Received 5 March 2013 / Accepted 10 October 2013

\begin{abstract}
Among the key parameters defining the interstellar media (ISM) of galaxies is the fraction of the metals that are locked up in dust: the metals-to-dust ratio. This ratio bears not only on the ISM and its evolution, but also particularly on the origin of cosmic dust. We combine extinction and abundance data from $\gamma$-ray burst (GRB) afterglows with similar data from quasar (QSO) foreground absorbers, as well as from multiply-imaged galaxy-lensed QSOs, to determine the metals-to-dust ratios for lines of sight through a wide diversity of galaxies from blue, dwarf starbursts to massive ellipticals, across a vast range of redshifts $z=0.1-6.3$, and nearly three orders of magnitude of column density and metal abundance. The GRB and lensed QSO extinction methods are the most reliable that are available outside the Local Group (LG), allowing absolute extinction measurements. We thus determine the metals-to-dust ratio in a unique way, providing direct determinations of in situ gas and dust columns without recourse to assumptions with large uncertainties. We find that the metals-to-dust ratios in these systems are surprisingly close to the value for the LG, with a mean value of $10^{21.2} \mathrm{~cm}^{-2} A_{V} \mathrm{mag}^{-1}$ and a standard deviation of $0.3 \mathrm{dex}$, compared to the Galactic value of $10^{21.3} \mathrm{~cm}^{-2} A_{V} \mathrm{mag}^{-1}$ (in units of the Galactic gas-to-dust ratio). There is no evidence of deviation from this mean ratio as a function of metallicity, even down to our lowest metallicity of $0.01 \mathrm{Z} / \mathrm{Z}_{\odot}$. The lack of any obvious dependence of the metals-to-dust ratio on column density, galaxy type or age, redshift, or metallicity indicates a close correspondence between the formation of the metals and the formation of dust. Any delay between the formation of metals and dust must be shorter than the typical metal-enrichment times of these galaxies, i.e. shorter than a few Myr. Formation of the bulk of the dust in low mass stars is therefore ruled out by these data at any cosmic epoch. Furthermore, dust destruction must not dominate over formation/growth in virtually any galaxy environment. The close correlation between metals and dust is a natural consequence of the formation of the bulk of cosmic dust in supernovae. Grain growth in the ISM, if it is to be the dominant cosmic dust formation mechanism, is strongly constrained by these data to operate on very short timescales.
\end{abstract}

Key words. galaxies: high-redshift - dust, extinction - gamma rays: general - quasars: general

\section{Introduction}

The important constituents of the interstellar medium (ISM) are gas, metals, and dust which play a crucial role in the properties of star formation and in the appearance, formation, and evolution of galaxies. Dust grains are made up of metals, primarily $\mathrm{O}$, $\mathrm{Si}, \mathrm{C}, \mathrm{Mg}$, and $\mathrm{Fe}$, which are introduced into the ISM by stars close to the end, or at the end of their lives. There is, however, significant debate over the origin of the bulk of the dust and, related to this, how quickly dust can form from these metals. This is an important issue from a cosmic perspective for a number of reasons. For example, fully half of the non-primordial radiation in the universe is reprocessed through dust grains, and among early bursts of star formation the presence of dust may have helped cool the gas in the formation of the second generation of stars (Schneider et al. 2012) and may have provided the catalyst for the formation of molecular hydrogen, the driver of star formation. The front-running candidates for the formation of the bulk of the dust are the cool, dense envelopes of evolved, low mass stars (Gail et al. 2009), supernova (SN) ejecta (Dunne et al. 2003), or grain growth in the dense ISM (Draine 2009). The observed metals-to-dust ratio and its evolution with total metallicity is a key parameter in tracing the origin of the bulk of the dust and how it evolves in the ISM. For example, if most of the dust is formed in $\mathrm{SNe}$ and is not largely destroyed thereafter, the metals-to-dust ratio should be fairly constant over time and be very similar in different types of galaxies. If, on the other hand, low-mass stellar envelopes form most of the dust, there should be a delay between the formation of metals and the formation of dust of a few billion years, which should be detectable in an apparent diversity and evolution of the metals-to-dust ratio.

In this paper we determine the metals-to-dust ratios for a large, diverse sample of galaxies as a function of total column density and metallicity. We use an absorption and extinction methodology to directly determine the metal and dust columns along individual lines of sight to produce metals-to-dust ratios that are far less prone to systematic and modeling uncertainties than those produced from emission measurements or from depletion estimates. Furthermore, our sample covers galaxies up to the edge of the reionization epoch, in a redshift range from $z=0.1-6.3$, and over an unprecedented metallicity range of $[\mathrm{M} / \mathrm{H}]=-2.0-0.5$.

We use extinctions and metal column densities from a large sample of $\gamma$-ray burst (GRB) afterglows, together with H I column densities where available. We also use gas and metal column densities from QSO foreground absorption systems together with extinctions derived from template fitting for these objects, and we use optical/UV extinctions and total metal column densities derived from X-ray spectroscopy for a few galaxylensed quasars. We compare these metals-to-dust ratios of GRB 
afterglows, QSO-damped Ly $\alpha$ absorbers (DLAs), and lensed galaxies to the value obtained in the Magellanic Clouds and in the Milky Way (MW).

In Sect. 2 we present our sample selection criteria and describe results from the analysis. In Sects. 3 and 4 we provide results and a brief discussion. The conclusions are summarized in Sect. 5. Throughout this paper we use cosmic abundances from Anders \& Grevesse (1989).

\section{Methods}

Previous studies of the metals-to-dust ratio outside the Local Group (LG) have relied mostly on emission measurements; from dust, from $\mathrm{HI}$, and a metallicity estimate from emission line ratios. These measurements presented a diversity of results depending on the methodology used and the assumptions made. The advantages of these studies are that they offer a total census of each galaxy to the sensitivity limits of the instrument or observation, and that the galaxies can often be resolved, allowing examination of the spatial distribution of ISM components. The inherent limitations of emission studies due to sensitivity limitations apply; the measurements of gas-phase metallicity do not cover the same region as the dust emission, which does not cover the same area as the $21 \mathrm{~cm} \mathrm{HI}$ emission; and luminous galaxies are more easily surveyed, and the studies can typically be conducted at low redshift. Furthermore, conversions from farinfrared (FIR) emission to dust mass require significant, uncertain assumptions even where a measurement of the dust temperature can be made.

The ISM of galaxies can also be pursued via absorption of strong backlighting sources. These sources are particularly useful where their intrinsic spectra are well known. And while only one specific sightline is observed through these galaxies, the advantages of working in absorption are numerous: a) essentially all stages of cosmic time are available, since some of these sources can be observed spectroscopically as far back as $z \sim 8$ (Tanvir et al. 2009); b) the method is highly sensitive, and even very low metallicities and dust contents are detectable; c) the observations always compare the same column of material, whether in dust, gas, or metals, meaning that there is no bias due to the relative sensitivity to these different components across a given galaxy; and d) the inherent luminosity of the absorber plays no role in its detection. Absorption spectroscopy in the restframe UV can also place direct limits on the column density of $\mathrm{H}_{2}$ (Krühler et al., in prep.; Prochaska et al. 2009; Noterdaeme et al. 2008). Gamma-ray burst afterglows and QSOs are the obvious backlighting candidates for this technique, as both are extremely luminous, have quite well-known spectra, and are thus excellent probes of absorbing gas and extinguishing dust along the line of sight. Most GRB afterglows are strongly absorbed by their own host galaxy's ISM (e.g. Vreeswijk et al. 2004; Fynbo et al. 2009; Watson et al. 2007) and so probe the ISM of strongly star-forming galaxies (Jakobsson et al. 2012) from $z=0.0086-$ 8.2, typically far away from the burst itself (Watson et al. 2007; Vreeswijk et al. 2004; D'Elia et al. 2009). Quasars, on the other hand, are used as probes of foreground galaxies along the line of sight, and so typically intersect the low-surface density outskirts of galaxies (e.g. Chen et al. 2005a). Measurements of the metalsto-dust ratio in QSO foreground absorbers have been made using the relative depletion of refractory elements out of the gas phase as a measurement of the dust column (Pei et al. 1999; Vladilo et al. 2006), rather than a direct extinction measure. Finally, QSOs are also occasionally strongly lensed and multiply imaged by the gravitational potential of a massive foreground galaxy.
Comparison of the two lensed images of these systems typically shows one image passing through the relatively dense ISM of the lensing galaxy, allowing the relative extinction and absorption properties of the two sightlines to be determined (e.g. Toft et al. 2000; Dai \& Kochanek 2009).

\subsection{Sample selection and analysis}

In a previous work we produced the largest sample of spectroscopic extinction curves to date outside the MW, using GRB afterglows (Zafar et al. 2011a). From this sample we have optical extinction estimates for 9 GRB afterglows. In addition, we have taken other GRBs from the literature with optical extinction, metal column density, and H I column density measurements, making up a total of 25 GRB afterglows. These additional GRBs were selected because they have their optical extinction derived from X-ray-to-optical/near-infrared spectral energy distribution fitting. To the GRB data we have added 17 QSO foreground absorbing systems and 6 gravitationally-lensed, multiply-imaged quasar systems. These are the ones we can find in the literature where both metal column density and reddening/extinction estimates are available (see Table 1 for details).

The extinction of GRBs is estimated from the deviation from a single or broken power-law of their X-ray-to-optical/nearinfrared spectral energy distribution. The extinction from lensed quasars is derived from the difference between the spectra of the multiple images. These two methods are the most reliable in determining extinctions at cosmological distances and can be used to determine absolute extinction curves. For QSO-DLAs reddening is measured either from QSO colors or based on template fitting, and so these values are less robust. The reddening template used is the Small Magellanic Cloud extinction curve of Pei (1992) except for one case, Q 1157+6135, where a MW-like extinction curve and a $2175 \AA$ bump is detected at the redshift of the DLA (Wang et al. 2012). To determine total metallicities and avoid biases related to depletion of metals from the gas phase onto dust, we derived metal column densities from nonrefractory elements, either $\mathrm{Zn}$ or $\mathrm{S}$ from low-ionization lines. To be consistent throughout, we used solar abundances from Anders \& Grevesse (1989) for metallicity determination. Anders \& Grevesse (1989) abundances (for oxygen in particular) are significantly higher than indicated by direct measurements of the solar spectrum (Asplund et al. 2009); however, they are probably a better estimate of the typical Galactic ISM abundance (see Watson 2011) and are the metallicities typically used in X-ray measurements to derive equivalent column densities of hydrogen. For GRBs 061121, 070506, 080319C, 080413B, 080605, and 080905B Zn equivalent widths from Fynbo et al. (2009) are used to obtain column densities using the optically thin approximation (see also Laskar et al. 2011). In order to be able to compare metal column densities, we derived a total equivalent column density for the GRB afterglows and QSO-DLAs by modifying $N_{\mathrm{HI}}$ for metallicity (i.e. $\left.\log \left(N_{\mathrm{HI}} / \mathrm{cm}^{-2}\right)+[\mathrm{M} / \mathrm{H}]\right)$. This then allows us to compare the total metal column density in a common reference, i.e. in terms of the equivalent gas column density for the Galaxy. Gamma-ray bursts 061121, 080319C $180413 \mathrm{~A}$, and 080605 have no $N_{\mathrm{HI}}$ estimates because they are at $z<2$. Therefore, their metallicities could not be derived. However, for these GRBs, the total metal column could be derived directly from the $\mathrm{S}$ or $\mathrm{Zn}$ column densities. For this analysis we also use results from galaxy-lensed quasars from Dai \& Kochanek (2009), where total metal column densities can be obtained from the soft X-ray absorption, something that is not 
Table 1. Basic data used to estimate metals-to-dust ratio.

\begin{tabular}{|c|c|c|c|c|c|c|}
\hline GRB & $\begin{array}{c}\log N_{\mathrm{HI}} \\
\mathrm{cm}^{-2}\end{array}$ & $\begin{array}{c}\log N_{\mathrm{X}} \\
\mathrm{cm}^{-2}\end{array}$ & $X$ & $\begin{array}{c}A_{V} \\
\text { mag }\end{array}$ & $z$ & Reference \\
\hline 000926 & $21.30 \pm 0.21$ & $13.82 \pm 0.05$ & $\mathrm{Zn}$ & $0.38 \pm 0.05$ & 2.038 & Chen et al. (2007), Starling et al. (2007) \\
\hline 030226 & $20.50 \pm 0.30$ & $<12.70$ & $\mathrm{Zn}$ & $0.05 \pm 0.01$ & 1.987 & Shin et al. (2006), Schady et al. (2011) \\
\hline 050401 & $22.60 \pm 0.30$ & $14.30 \pm 0.30$ & $\mathrm{Zn}$ & $0.65 \pm 0.04$ & 2.899 & Watson et al. (2006), Zafar et al. (2011a) \\
\hline 050505 & $22.05 \pm 0.10$ & $>16.10$ & $\mathrm{~S}$ & $0.30 \pm 0.1$ & 4.275 & Berger et al. (2006), Hurkett et al. (2006) \\
\hline 050730 & $22.15 \pm 0.06$ & $15.34 \pm 0.10$ & $\mathrm{~S}$ & $0.12 \pm 0.02$ & 3.969 & Chen et al. (2005b), Zafar et al. (2011a) \\
\hline 050820A & $21.05 \pm 0.10$ & $13.28 \pm 0.04$ & $\mathrm{Zn}$ & $0.27 \pm 0.04$ & 2.612 & Ledoux et al. (2009), Schady et al. (2012) \\
\hline 050904 & $21.62 \pm 0.02$ & $15.14 \pm 0.17$ & $\mathrm{~S}$ & $<0.05$ & 6.295 & Thöne et al. (2013), Zafar et al. (2010, 2011b) \\
\hline 050922C & $21.55 \pm 0.10$ & $14.92 \pm 0.05$ & $S$ & $<0.24$ & 2.198 & Schady et al. (2012) \\
\hline 060206 & $20.85 \pm 0.10$ & $15.13 \pm 0.05$ & S & $<0.23$ & 4.048 & Thöne et al. (2008), Schady et al. (2012) \\
\hline 060526 & $20.00 \pm 0.15$ & $14.58 \pm 0.25$ & $\mathrm{~S}$ & $<0.39$ & 3.221 & Thöne et al. (2010), Schady et al. (2011) \\
\hline 061121 & $\ldots$ & $13.76 \pm 0.06$ & $\mathrm{Zn}$ & $0.55 \pm 0.10$ & 1.315 & Schady et al. (2012) \\
\hline 070506 & $22.00 \pm 0.30$ & $>13.68$ & $\mathrm{Zn}$ & $0.44 \pm 0.05$ & 2.308 & Fynbo et al. (2009), Zafar et al. (2011a) \\
\hline 070802 & $21.50 \pm 0.20$ & $13.60 \pm 0.60$ & $\mathrm{Zn}$ & $1.19 \pm 0.15$ & 2.455 & Elíasdóttir et al. (2009), Zafar et al. (2011a) \\
\hline 071031 & $22.15 \pm 0.05$ & $13.05 \pm 0.03$ & $\mathrm{Zn}$ & $<0.07$ & 2.692 & Ledoux et al. (2009), Zafar et al. (2011a) \\
\hline 080210 & $21.90 \pm 0.10$ & $13.53 \pm 0.14$ & $\mathrm{Zn}$ & $0.33 \pm 0.03$ & 2.641 & De Cia et al. (2011), Zafar et al. (2011a) \\
\hline 080319C & $\ldots$ & $13.64 \pm 0.60$ & $\mathrm{Zn}$ & $0.67 \pm 0.07$ & 1.949 & Perley et al. (2009) \\
\hline 080413A & $21.85 \pm 0.15$ & $12.88 \pm 0.07$ & $\mathrm{Zn}$ & $<0.59$ & 2.433 & Ledoux et al. (2009), Schady et al. (2011) \\
\hline 080413B & $\ldots$ & $13.57 \pm 0.15$ & $\mathrm{Zn}$ & $0.84 \pm 0.16$ & 1.101 & Schady et al. (2012) \\
\hline 080605 & $\cdots$ & $13.54 \pm 0.30$ & $\mathrm{Zn}$ & $0.50 \pm 0.13$ & 1.640 & Zafar et al. (2012) \\
\hline 080607 & $22.70 \pm 0.15$ & $>16.34$ & $\mathrm{~S}$ & $2.33_{-0.43}^{+0.46}$ & 3.037 & Prochaska et al. (2009), Zafar et al. (2011a) \\
\hline 080905B & $<22.15$ & $13.52 \pm 0.13$ & $\mathrm{Zn}$ & $0.42 \pm 0.03$ & 2.374 & Fynbo et al. (2009), Zafar et al. (2011a) \\
\hline 081008 & $21.11 \pm 0.10$ & $13.15 \pm 0.04$ & $\mathrm{Zn}$ & $\approx 0.19$ & 1.968 & D’Elia et al. (2011) \\
\hline 090323 & $19.62 \pm 0.33$ & $15.41 \pm 0.04$ & $\mathrm{~S}$ & $0.10 \pm 0.04$ & 3.577 & Savaglio et al. (2012), Schady et al. (2011) \\
\hline 090926A & $21.60 \pm 0.07$ & $14.89 \pm 0.10$ & S & $<0.03$ & 2.107 & D'Elia et al. (2010) \\
\hline $100219 \mathrm{~A}$ & $21.14 \pm 0.15$ & $15.25 \pm 0.15$ & $S$ & $0.13 \pm 0.05$ & 4.667 & Thöne et al. (2013) \\
\hline QSO & $\log N_{\mathrm{HI}}$ & $\log N_{\mathrm{X}}$ & $\mathrm{X}$ & $A_{V}$ & $z$ & Ref. \\
\hline $0013+0004$ & $20.80 \pm 0.01$ & $12.25 \pm 0.05$ & $\mathrm{Zn}$ & $<0.10$ & 2.025 & Vladilo et al. (2006) \\
\hline 0016-0012 & $20.83 \pm 0.05$ & $12.82 \pm 0.04$ & $\mathrm{Zn}$ & $0.16_{-0.06}^{+0.04}$ & 1.973 & Vladilo et al. (2006) \\
\hline $0121+0027$ & $\ldots$ & $>13.32$ & $\mathrm{Zn}$ & $0.69_{-0.2}^{+3.2}$ & 1.388 & Vladilo et al. (2006) \\
\hline $0816+1446$ & $22.00 \pm 0.10$ & $13.53 \pm 0.01$ & $\mathrm{Zn}$ & $<0.50$ & 3.287 & Guimarães et al. (2012) \\
\hline $0918+1636$ & $20.96 \pm 0.05$ & $13.40 \pm 0.01$ & $\mathrm{Zn}$ & $\approx 0.21$ & 2.580 & Fynbo et al. (2011) \\
\hline $0938+4128$ & $20.52 \pm 0.10$ & $12.25 \pm 0.05$ & $\mathrm{Zn}$ & $<0.20$ & 1.373 & Vladilo et al. (2006) \\
\hline $0948+4323$ & $21.62 \pm 0.06$ & $13.15 \pm 0.01$ & $\mathrm{Zn}$ & $<0.31$ & 1.233 & Vladilo et al. (2006) \\
\hline $1010+0003$ & $21.52 \pm 0.07$ & $13.15 \pm 0.06$ & $\mathrm{Zn}$ & $<0.13$ & 1.265 & Vladilo et al. (2006) \\
\hline $1107+0048$ & $20.98 \pm 0.15$ & $13.03 \pm 0.05$ & $\mathrm{Zn}$ & $<0.26$ & 0.741 & Vladilo et al. (2006) \\
\hline $1157+6135$ & $21.80 \pm 0.20$ & $\approx 13.80$ & $\mathrm{Zn}$ & $0.92 \pm 0.07$ & 2.459 & Wang et al. (2012) \\
\hline $1159+0112$ & $21.80 \pm 0.10$ & $13.09 \pm 0.08$ & $\mathrm{Zn}$ & $0.14_{-0.06}^{+0.04}$ & 1.944 & Vladilo et al. (2006) \\
\hline $1232-0224$ & $20.75 \pm 0.07$ & $12.93 \pm 0.12$ & $\mathrm{Zn}$ & $<0.32$ & 0.395 & Vladilo et al. (2006) \\
\hline $1237+0647$ & $20.00 \pm 0.15$ & $13.02 \pm 0.02$ & $\mathrm{Zn}$ & $0.15 \pm 0.03$ & 2.690 & Noterdaeme et al. (2010) \\
\hline $1323-0021$ & $20.21 \pm 0.20$ & $13.43 \pm 0.05$ & $\mathrm{Zn}$ & $0.44_{-0.11}^{+0.08}$ & 0.716 & Vladilo et al. (2006) \\
\hline $1501+0019$ & $20.85 \pm 0.05$ & $13.10 \pm 0.05$ & $\mathrm{Zn}$ & $<0.16$ & 1.483 & Vladilo et al. (2006) \\
\hline $2234+0000$ & $20.56 \pm 0.10$ & $12.46 \pm 0.02$ & $\mathrm{Zn}$ & $<0.25$ & 2.066 & Vladilo et al. (2006) \\
\hline $2340-0053$ & $\ldots$ & $12.62 \pm 0.05$ & $\mathrm{Zn}$ & $0.21_{-0.11}^{+0.06}$ & 1.360 & Vladilo et al. (2006) \\
\hline Lensed QSO & $\log N_{\mathrm{HI}}$ & $\log N_{\mathrm{X}}$ & $\mathrm{X}$-ray & $A_{V}{ }^{a}$ & $z$ & Ref. \\
\hline SBS $0909+523$ & $\ldots$ & $20.78_{-0.20}^{+0.42}$ & X-ray & $0.99 \pm 0.03$ & 0.830 & Dai \& Kochanek (2009) \\
\hline В $1152+199$ & $\ldots$ & $21.68_{-0.04}^{+0.04}$ & X-ray & $3.72 \pm 0.16$ & 0.439 & Dai \& Kochanek (2009) \\
\hline MG 0414+0534 & $\cdots$ & $21.52_{-0.18}^{+0.12}$ & X-ray & $0.56 \pm 0.34$ & 0.958 & Dai \& Kochanek (2009) \\
\hline B $1600+434$ & $\ldots$ & $21.42_{-0.27}^{+0.21}$ & X-ray & $0.31 \pm 0.09$ & 0.410 & Dai \& Kochanek (2009) \\
\hline PKS 1830-211 & $\ldots$ & $22.25_{-0.20}^{+0.12}$ & X-ray & $9.30 \pm 0.40$ & 0.886 & Dai \& Kochanek (2009) \\
\hline Q 2237+0305 & $\cdots$ & $20.60_{-0.60}^{+0.24}$ & X-ray & $0.34 \pm 0.09$ & 0.040 & Dai \& Kochanek (2009) \\
\hline
\end{tabular}

Notes. The details are provided in columns as (1) GRB name; (2) $\log N_{\mathrm{Hi}}$; (3) metal column densities; (4) reference element either X=Zn or S or soft X-ray; (5) optical extinction; (6) redshift; and (7) references. $2 \sigma$ upper limits are provided for $A_{V}$ non-detections. ${ }^{(a)}$ For $R_{V}=3.1$. 


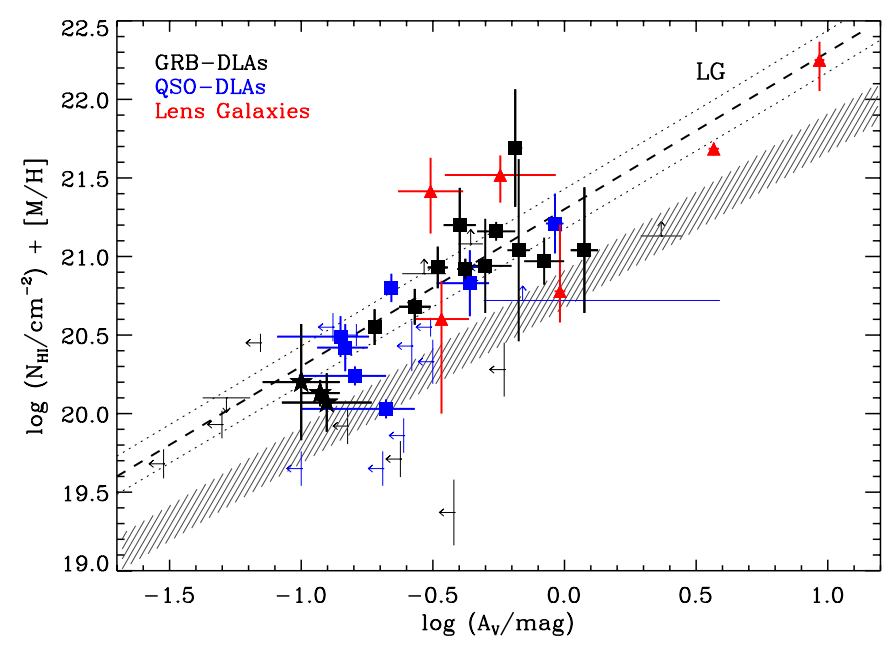

Fig. 1. Equivalent metal column density; $N_{\mathrm{HI}}$ corrected for metallicity in GRB afterglows (black), QSO-DLAs (blue), and nearby lensed galaxies (red) from Dai \& Kochanek (2009, black) as a function of dust extinction. The dashed curve represents the metals-to-dust ratio for the LG environments. The dotted lines are metals-to-dust ranges for the MW. The Zn-based metallicities are shown by squares while the stars represent the S-based metallicities. The gray shaded area represents the lower bound below which objects probably cannot exist (see text).

possible for long GRBs because of their anomalous X-ray column densities (Watson et al. 2013), again using the same abundances. The metallicities of lensed quasars are not derived because of the lack of $N_{\mathrm{HI}}$ column density estimates.

\section{Results}

In Fig. 1 we show the optical extinction and equivalent metal column density for GRB afterglows and QSO-DLAs. In this comparison, we span more than three orders of magnitude in column density and include a very diverse range of objects, everything from massive ellipticals (Toft et al. 2000), to the hearts of starforming galaxies (Watson et al. 2007) and to the outskirts of galaxies (Chen et al. 2005a; Ellison et al. 2005). Furthermore, our results span very large redshift $(z=0.1-6.3)$ and metallicity ranges $([\mathrm{M} / \mathrm{H}]=-2.0-0.5)$. We compared all these results to the metals-to-dust relation for the Galaxy and Magellanic Clouds (here referred to as LG). For the LG, we use here a canonical value of metals-to-dust ratio $\approx 2 \times 10^{21} \mathrm{~cm}^{-2} \mathrm{mag}^{-1}$ (see Watson 2011, and references therein).

Systems significantly below the LG relation are not expected, since a very large fraction of refractory metals are already in the dust phase in the Galaxy and Magellanic Clouds (Draine 2003). Practically, systems with less than about half the metals-to-dust ratio of the LG cannot exist, as there are simply not enough metals available to form more dust. Comparing the LG metals-to-dust ratio with the mean LG metals-to-dust mass ratio (i.e. 2.3, Pei et al. 1999, and references therein), we have shaded the region in Fig. 1 below which objects cannot exist for this reason. On the other hand, objects that are very metalrich and at the same time have relatively low $A_{V}$ (top-left region of Fig. 1) should be easy to find if they existed. However, we find no cases that are significantly dust-poor with respect to the amount of metals present compared to the $\mathrm{LG}$ values. We cover a very wide range in column density, but we see almost the same metals-to-dust ratio everywhere and centered at the LG relation. The tightness of this relation across so many different environments, redshifts, metallicities, and column densities is quite surprising. For our data, we find a mean metals-to-dust ratio of $10^{21.2} \mathrm{~cm}^{-2} A_{V}^{-1}$ (detections only) for our assumed abundances, with a standard deviation of 0.3 dex. Including limits in the analysis, we find a mean metals-to-dust ratio of $10^{21.1} \mathrm{~cm}^{-2} A_{V}^{-1}$, with a standard deviation of 0.4 dex. Previously Zafar et al. (2011a) found that GRBs have slightly higher metals-to-dust ratios compared to the LG using UV absorption lines. The UV line metallicities in that case were derived based on the solar abundances of Asplund et al. (2009). The metals-to-dust ratio was compared to the LG value based on Galactic X-ray data, which almost invariably uses the solar abundance set of Anders \& Grevesse (1989), because it is representative of typical Galactic ISM abundances (Watson 2011). In the present work we use $S$ and $\mathrm{Zn}$ based metallicities, but adopt a set of solar abundances to make the comparison consistent between the X-ray derived, LG, metals-to-dust ratio, and the UV line column densities (in this case Anders \& Grevesse 1989). We find that the metals-to-dust ratio is consistent with the LG relation.

Foreground absorbers may add some contribution to the observed extinction. Ménard et al. (2008) have determined a color excess that decreases strongly with redshift $(E(B-V) \propto$ $\left.(1+z)^{-1.1}\right)$ for their foreground galaxies. Using their results, the correction will typically be $A_{V}<0.05$ for our QSO-DLAs and GRBs, decreasing to high redshift, and is thus expected to be within or close to the level of our uncertainties in most cases. Our most constraining datapoint, GRB 050730 with $[\mathrm{M} / \mathrm{H}]=-2.02$, is worth a special note. Because of its especially low extinction $\left(A_{V}=0.12 \pm 0.02\right)$, it may be vulnerable to intervening absorption. We note that it has some intervening foreground absorbers, at $z=3.564,2.262,2.253$, and 1.772 (D'Elia et al. 2007). Using the relation of Ménard et al. (2008), we estimate that approximately one third of the observed extinction should be due to known foreground absorbers. This correction would make the data more consistent with the LG relation. As we noted above, the mean line of sight should not be heavily affected by intervening absorbers. However, given the dependence of our result at low metallicities (below $[\mathrm{M} / \mathrm{H}]<-1.3$ ) on this single datapoint, one should be cautious about whether foreground absorbers at lower redshifts $(z \lesssim 1.2)$ could contribute in this specific case, since most of the flux shortward of $6200 \AA$ is removed by hydrogen Lyman absorption.

\subsection{Metals-to-dust ratio versus metallicity}

One of the key diagnostics of where and when dust is formed is its variation with metallicity. Our GRB (and QSO-DLA) data are rich enough that we can determine metallicities for our sample above $z \sim 1.8$ (due to the atmospheric window for H I Ly $\alpha$ ). We therefore show how the metals-to-dust ratio varies as a function of metallicity for these objects in Fig. 2. There is no evidence of any variation in the metals-to-dust ratio with metallicity, even objects with metallicities as low as $\sim 1 \%$ of the solar metallicity show no indication of a different metals-to-dust ratio. Finally, we show the metals-to-dust ratio as a function of redshift (Fig. 3), and again, we find no apparent change, i.e. the metals-to-dust ratio we find at $z \sim 6.3$ is the same as in our own Galaxy.

Pei et al. (1999) estimated dust masses by measuring the depletion of $[\mathrm{Cr} / \mathrm{Zn}]$ and reported that the mean dust-to-metals ratio was roughly constant over a redshift range $0 \lesssim z \lesssim 3$. In the present study, we are measuring the full dust column directly through extinction (GRBs) or reddening (QSOs) rather than inferring its presence from the relative depletion of $\mathrm{Cr}$. Our data is consistent with the results found by Pei et al. (1999) but extends 


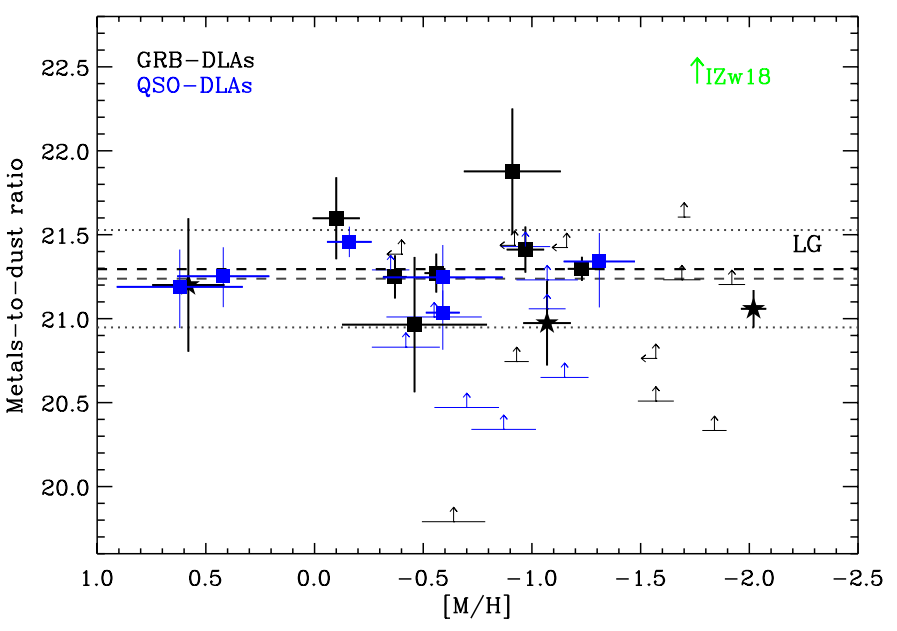

Fig. 2. Metals-to-dust ratio $\left(\left(\log N_{\mathrm{HI}} / \mathrm{cm}^{-2}+[\mathrm{M} / \mathrm{H}]\right)-\log A_{V} / \mathrm{mag}\right)$ versus metallicity for GRB afterglows (black) and QSO-DLAs (blue). The black dashed line represents the LG metals-to-dust relation. The gray dashed line is the mean metals-to-dust ratio of our sample and standard deviation is illustrated by dotted lines. The symbols have the same meaning as in Fig. 1. For comparison, the upper limit derived by Herrera-Camus et al. (2012) from SED modeling of the lowest metallicity galaxy known in the local universe, IZw 18 , is plotted in green.

the redshift range from $z<3$ to $z>6$, and covers a much wider variety of objects, dealing not only with QSO-DLAs, but also with the hearts of star-forming galaxies.

\section{Discussion}

The origin of cosmic dust is currently a major issue in astrophysics and cosmology. The three candidates for forming the bulk of the dust are condensation in the envelopes of low mass, evolved stars (AGB dust formation, Gail et al. 2009), condensation in SN ejecta (Dunne et al. 2003), and growth in dense molecular clouds (Draine 2009). While it has long been advocated on the basis of models of dust formation in AGB stars and dust destruction in the ISM that AGB stars are not sufficient to explain all of the dust in the Galaxy (e.g. Draine \& Salpeter 1979; Gehrz 1989), they dominate the pre-solar grain populations found to date (Nittler 2009), and were believed to be the dominant form of dust from stars (Gail et al. 2009). Furthermore, it has been proposed that they were major contributors to dust in the early universe (Valiante et al. 2009; Cherchneff \& Dwek 2009). Conversely, Maiolino et al. (2004), Dwek et al. (2007; 2011), Michałowski et al. (2010), Gall et al. (2011a,b), claimed that AGB stars are not efficient enough at high redshifts and that dust is due to SNe or ISM dust grain growth.

For dominant dust formation by AGB stars, there is a significant time delay between the formation of the bulk of the metals (which are formed in $\mathrm{SNe}$ ) and the bulk of the dust, because of the time required to evolve off the main sequence for these stars. This delay will introduce significant scatter in the metals-to-dust ratio, particularly showing deviations at the lowest metallicities, where the metals-to-dust ratios should be very high, since the dust has typically not had time to form.

For bulk dust formation via accretion of dust onto seed particles in the dense ISM, the growth rate is strongly related to the metallicity of the ISM. Indeed, in most analyses there is a critical metallicity below which grain growth in molecular

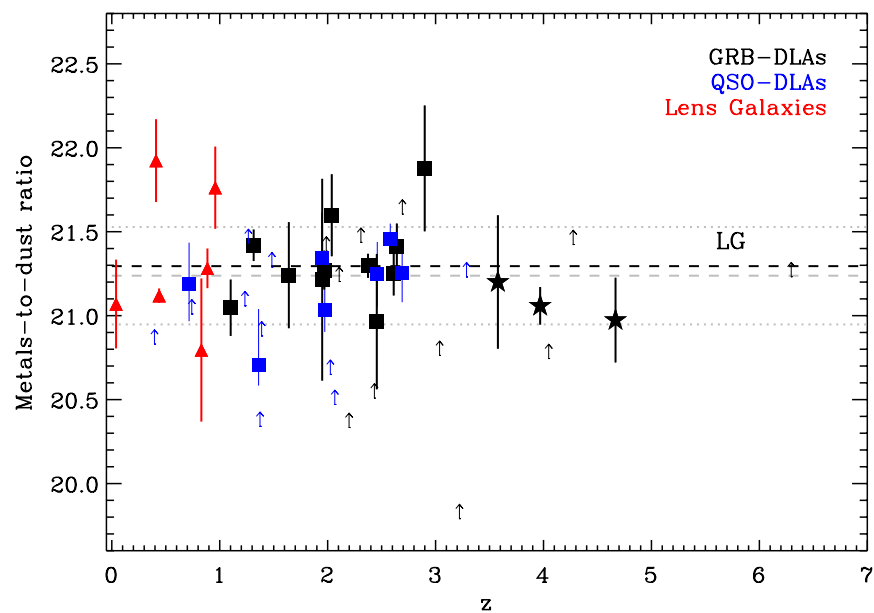

Fig. 3. Metals-to-dust ratio versus redshift for GRB afterglows (black), QSO-DLAs (blue), and lensed galaxies (red). The black dashed line represents the LG metals-to-dust relation. The gray dashed line is the mean metals-to-dust ratio of our sample and its standard deviation is illustrated by dotted lines. The symbols have the same meaning as in Fig. 1.

clouds is likely to be insignificant (e.g. Zhukovska \& Gail 2009; Asano et al. 2011). The precise critical metallicity is dependent on the sticking coefficient for the grains, the survival times of the molecular clouds, and the injected grain size distribution. Zhukovska \& Gail (2009) indicate that grain growth in molecular clouds only becomes dominant above a metallicity of $[\mathrm{M} / \mathrm{H}] \sim-1$. Again, this indicates that if dust formation is dominated by this mechanism, there should be a fall away from a constant metals-to-dust ratio at low metallicities. Where precisely this low metallicity value lies is unclear. However, down to $[\mathrm{M} / \mathrm{H}]=-2$, we see no sign of it in our data (Fig. 2). If grain growth in the ISM does dominate dust masses, the process must therefore be much more efficient than assumed in Zhukovska \& Gail (2009), implying an extremely fast dust growth timescale in higher metallicity systems. Draine (2009) makes a simple, order of magnitude estimate of the timescale required for dust growth in the ISM. According to Eq. (8) of Draine (2009), a 1\% metallicity would imply an accretion timescale of $1 \mathrm{Gyr}$, far longer than the typical stellar ages of GRB hosts (Christensen et al. 2004; Savaglio et al. 2009; Jakobsson et al. 2012), and therefore inconsistent with our data. On the other hand, charged polycyclic aromatic hydrocarbons (PAH) could make the initial growth rate a factor of 50 faster than this (Draine 2009) at least for the growth of the smallest grain, i.e. $20 \mathrm{Myr}$, which is consistent with the typical stellar ages of GRB hosts. However, we should note that this is extreme, since it relies on PAH growth, which seems unlikely to drive the bulk of grain growth. Future measurements of dust and metal columns in objects with metallicities significantly below $1 \%$ of the solar abundance should thus distinguish between ISM grain growth and SN-formation (see below) as the dominant dust-making scenario. As a corollary, this analysis also implies that in solar metallicity systems, the grain accretion timescales are typically less than 1 Myr. A similarly short timescale has been inferred on other grounds based on an analysis of dusty high redshift galaxies (Watson et al., in prep.; see also Calura et al. 2008; Pipino et al. 2011).

Finally, a constant metals-to-dust ratio is a natural consequence of formation of the bulk of the dust in core collapse $\mathrm{SNe}$. In this scenario, if core-collapse $\mathrm{SNe}$ turn most of their 
refractory metals into dust, as long as there is not wholesale return of the metals to the gas via destruction of dust in the ISM, then a constant metals-to-dust ratio is what would be expected across all redshifts, metallicities, and galaxy types.

\subsection{Comparison with other studies}

We cover approximately 2.5 orders of magnitude in metallicity and find that the ratio in both metal-rich and metal-poor systems is not only constant, but also that the mean is consistent with the values found for the Galaxy and Magellanic Clouds. We do not find any significant variation in the metals-to-dust ratios of systems with $[\mathrm{M} / \mathrm{H}] \lesssim-1.5$. This hints that metal-poor and metalrich galaxies form dust in the same way. Draine et al. (2007) show that when considering only the spatial regions over which the dust is detected, the metals-to-dust ratios appear to be constant for the Spitzer Infrared Nearby Galaxies Survey (SINGS) galaxies, a result consistent with what we find here, though subject to greater uncertainties, and covering a far less diverse sample in both metallicity and cosmic age.

The most sensitive data in this connection, of course, are very low metallicity systems, for the reasons mentioned above. Chemical evolution models considering dust destruction by SNe (Hirashita et al. 2002) or mass outflows from the galaxy (Lisenfeld \& Ferrara 1998) predict that at low metallicities the gas-to-dust ratio should not scale linearly with metallicity as it seems to at higher metallicities. In examining dwarf, metalpoor galaxies, Galametz et al. (2011) showed that the addition of submm data to SED modeling was important and resulted in higher dust masses than when modeled without submm data, and showed once again a tight correlation of the dust-to-gas mass ratio with metallicity. In searching for such low metallicity systems, emission studies have turned to the most metal-poor system known in the local Universe, the blue compact dwarf galaxy IZw 18 (Herrera-Camus et al. 2012) with $[\mathrm{O} / \mathrm{H}]=-1.76$ (using Anders \& Grevesse (1989) abundances). For IZw 18, HerreraCamus et al. (2012) find a higher gas-to-dust ratio compared to the available metallicity, suggesting that at low metallicities the dust fraction may fall. We plot their suggested metals-to-dust ratio in Fig. 2 for comparison to our data, but it is worth noting that they assume a constant metallicity across the galaxy, and have some uncertainty in their determination of the total dust mass, since they do not detect cool dust emission, and since the dust mass is highly dependent on the assumed dust properties, temperature, and dust distribution. Using only the same spatial regions (cf. Draine et al. 2007), and one of the dust models, Herrera-Camus et al. (2012) note that the IZw 18 dust-togas ratio is only a factor of two below that expected for the metallicity, which is within the scatter observed in the Draine et al. (2007) sample. It is therefore unclear so far whether emission measurements indicate a significant change in the metalsto-dust ratio at low metallicity. Future observations with the Atacama Large Millimeter/submillimeter Array (ALMA) should help resolve that debate within the uncertainties of emission diagnostics.

Other observations of the metals-to-dust ratio or gas-to-dust ratio as a function of metallicity include measurements using many different techniques in the Galaxy (see Watson 2011, and references therein), in the Magellanic Clouds (Weingartner \& Draine 2001; Gordon et al. 2003; Bernard et al. 2008), as well as in nearby spiral galaxies (Issa et al. 1990) and dwarf galaxies (Lisenfeld \& Ferrara 1998). Recently Smith et al. (2012) reported that the dust-to-gas ratio gradient of the Andromeda (M31) galaxy varies radially, consistent with its metallicity gradient. All of these measurements are consistent with our findings here that indicate a universal metals-to-dust ratio constant to within a factor of $30-40 \%$ (see also Dai \& Kochanek 2009, as well as a recent more expanded sample by Chen et al. 2013, again consistent with our conclusions here). We thus argue that the metals-to-dust ratio is constant within this scatter, i.e. 0.3 dex, down to $1 \%$ solar metallicity and suggest that the modeling uncertainties associated with emission measures are such that the current data are consistent with this conclusion.

Apart from the study of Pei et al. (1999) mentioned in Sect. 3.1, Noterdaeme et al. (2008) have used the depletions of a sample of QSO absorbers to investigate the dust-to-metals ratios as a function of metallicity and redshift. They found a correlation between the depletion of metals and the metallicity, using principally the depletion of iron-group elements. Their results indicate that fractionally less Fe is taken up in metals as the metallicity drops. Very recently, (De Cia et al. 2013) have demonstrated the same effect in GRB host galaxy absorbers. These results appear to contradict our findings here, and may be at odds with those of the SINGS sample (Draine 2009), which do not show a consistent rise in the metals-to-dust ratio with metallicity. A possible explanation of this may be that Fe does not dominate the dust mass, is synthesised in largely different locations, and appears to be depleted at a very different rate out of the gas phase, compared to the principal dust constituents, $\mathrm{Mg}, \mathrm{Si}, \mathrm{O}$, and $\mathrm{C}$ (Jenkins 2009). It is possible therefore that the depletion of $\mathrm{Fe}$ is not representative of the total dust column. Strong evidence that the dust column follows the Fe depletion would therefore be valuable. Our estimates of $A_{V}$ are somewhat dependent on the slopes of the extinction curves for GRBs which are often poorly constrained where we do not have infrared data. Our planned analysis of afterglows observed with X-shooter will be valuable in this respect. Finally, our results here at the lowest metallicity are subject to the possible caveat on foreground absorbers toward GRB 050730 mentioned in Sect. 3.

\section{Conclusions}

In this work we presented the metals-to-dust ratios of a sample of GRB afterglows as a function of their metallicities and redshifts. We supplemented this sample with QSO-DLAs, and lensed QSOs. Our data span 3 orders of magnitude in column density and 2.5 in metallicity. The redshifts range from $z=0.1$ to $z=6.3$, and galaxies vary in type from blue, sub-luminous starforming galaxies to massive ellipticals. We found the metalsto-dust ratio to be consistent with the LG relation and constant within a factor of 30-40\% regardless of metallicity, galaxy type, total column density, or redshift. We found that the metals-todust ratio of our sample is always close to the value for the LG (i.e. $10^{21.3} \mathrm{~cm}^{-2} A_{V}^{-1}$ ) for all of these systems, with a mean value of $10^{21.2} \mathrm{~cm}^{-2} A_{V}^{-1}$ and a standard deviation of $0.3 \mathrm{dex}$. This provides clear evidence of a near-universal metals-to-dust ratio within this scatter down to very low metallicity and in the early universe. We infer from this that dust formation closely follows metal formation with at most a short time delay ( $\lesssim 1 \mathrm{Myr})$ between them. The most natural interpretation of the data are that core collapse SNe produce the bulk of both the metals and the dust simultaneously. The data essentially rule out low mass stars as the origin of the bulk of the dust mass at any cosmic epoch, and puts strong constraints on models of dust growth in the dense ISM. 
Acknowledgements. This work has been funded within the BINGO! ("history of Baryons: INtergalactic medium/Galaxies cO-evolution") project by the Agence Nationale de la Recherche (ANR) under the allocation ANR-08-BLAN-0316-01. The Dark Cosmology Centre is funded by the Danish National Research Foundation. We are thankful to Jens Hjorth, Anja Andersen, Lars Mattsson, Thomas Krühler, and Annalisa De Cia for helpful comments. We are thankful to the GRB and QSO community for observing these remarkable objects and providing a well sampled data set.

\section{References}

Anders, E., \& Grevesse, N. 1989, Geochim. Cosmochim. Acta, 53, 197

Asano, R. S., Takeuchi, T. T., Hirashita, H., \& Inoue, A. K. 2011, in Why Galaxies Care about AGB Stars II: Shining Examples and Common Inhabitants, eds. F. Kerschbaum, T. Lebzelter, \& R. F. Wing, ASP Conf. Ser., 445,523

Asplund, M., Grevesse, N., Sauval, A. J., \& Scott, P. 2009, ARA\&A, 47, 481

Berger, E., Penprase, B. E., Cenko, S. B., et al. 2006, ApJ, 642, 979

Bernard, J.-P., Reach, W. T., Paradis, D., et al. 2008, AJ, 136, 919

Calura, F., Pipino, A., \& Matteucci, F. 2008, A\&A, 479, 669

Chen, H.-W., Kennicutt, Jr., R. C., \& Rauch, M. 2005a, ApJ, 620, 703

Chen, H.-W., Prochaska, J. X., Bloom, J. S., \& Thompson, I. B. 2005b, ApJ, 634, L25

Chen, H.-W., Prochaska, J. X., Ramirez-Ruiz, E., et al. 2007, ApJ, 663, 420

Chen, B., Dai, X., Kochanek, C. S., \& Chartas, G. 2013, ApJ, submitted [arXiv: 1306.0008]

Cherchneff, I., \& Dwek, E. 2009, ApJ, 703, 642

Christensen, L., Hjorth, J., \& Gorosabel, J. 2004, A\&A, 425, 913

Dai, X., \& Kochanek, C. S. 2009, ApJ, 692, 677

De Cia, A., Jakobsson, P., Björnsson, G., et al. 2011, MNRAS, 412, 2229

De Cia, A., Ledoux, C., Savaglio, S., Schady, P., \& Vreeswijk, P. M. 2013, A\&A, in press, DOI: 10.1051/0004-6361/201321834

D'Elia, V., Fiore, F., Meurs, E. J. A., et al. 2007, A\&A, 467, 629

D'Elia, V., Fiore, F., Perna, R., et al. 2009, ApJ, 694, 332

D'Elia, V., Fynbo, J. P. U., Covino, S., et al. 2010, A\&A, 523, A36

D'Elia, V., Campana, S., Covino, S., et al. 2011, MNRAS, 418, 680

Draine, B. T. 2003, ARA\&A, 41, 241

Draine, B. T. 2009, in Cosmic Dust - Near and Far, eds. T. Henning, E. Grün, \& J. Steinacker, ASP Conf. Ser., 414, 453

Draine, B. T., \& Salpeter, E. E. 1979, ApJ, 231, 438

Draine, B. T., Dale, D. A., Bendo, G., et al. 2007, ApJ, 663, 866

Dunne, L., Eales, S., Ivison, R., Morgan, H., \& Edmunds, M. 2003, Nature, 424, 285

Dwek, E., Galliano, F., \& Jones, A. P. 2007, ApJ, 662, 927

Dwek, E., Staguhn, J. G., Arendt, R. G., et al. 2011, ApJ, 738, 36

Elíasdóttir, Á., Fynbo, J. P. U., Hjorth, J., et al. 2009, ApJ, 697, 1725

Ellison, S. L., Kewley, L. J., \& Mallén-Ornelas, G. 2005, MNRAS, 357, 354

Fynbo, J. P. U., Jakobsson, P., Prochaska, J. X., et al. 2009, ApJS, 185, 526

Fynbo, J. P. U., Ledoux, C., Noterdaeme, P., et al. 2011, MNRAS, 413, 2481

Gail, H.-P., Zhukovska, S. V., Hoppe, P., \& Trieloff, M. 2009, ApJ, 698, 1136

Galametz, M., Madden, S. C., Galliano, F., et al. 2011, A\&A, 532, A56

Gall, C., Andersen, A. C., \& Hjorth, J. 2011a, A\&A, 528, A13

Gall, C., Andersen, A. C., \& Hjorth, J. 2011b, A\&A, 528, A14

Gehrz, R. 1989, in Interstellar Dust, eds. L. J. Allamandola, \& A. G. G. M.

Tielens, IAU Symp., 135, 445
Gordon, K. D., Clayton, G. C., Misselt, K. A., Landolt, A. U., \& Wolff, M. J. 2003, ApJ, 594, 279

Guimarães, R., Noterdaeme, P., Petitjean, P., et al. 2012, AJ, 143, 147

Herrera-Camus, R., Fisher, D. B., Bolatto, A. D., et al. 2012, ApJ, 752, 112

Hirashita, H., Tajiri, Y. Y., \& Kamaya, H. 2002, A\&A, 388, 439

Hurkett, C. P., Osborne, J. P., Page, K. L., et al. 2006, MNRAS, 368, 1101

Issa, M. R., MacLaren, I., \& Wolfendale, A. W. 1990, A\&A, 236, 237

Jakobsson, P., Hjorth, J., Malesani, D., et al. 2012, ApJ, 752, 62

Jenkins, E. B. 2009, ApJ, 700, 1299

Laskar, T., Berger, E., \& Chary, R.-R. 2011, ApJ, 739, 1

Ledoux, C., Vreeswijk, P. M., Smette, A., et al. 2009, A\&A, 506, 661

Lisenfeld, U., \& Ferrara, A. 1998, ApJ, 496, 145

Maiolino, R., Schneider, R., Oliva, E., et al. 2004, Nature, 431, 533

Ménard, B., Nestor, D., Turnshek, D., et al. 2008, MNRAS, 385, 1053

Michałowski, M. J., Murphy, E. J., Hjorth, J., et al. 2010, A\&A, 522, A15

Nittler, L. R. 2009, PASA, 26, 271

Noterdaeme, P., Ledoux, C., Petitjean, P., \& Srianand, R. 2008, A\&A, 481, 327

Noterdaeme, P., Petitjean, P., Ledoux, C., et al. 2010, A\&A, 523, A80

Pei, Y. C. 1992, ApJ, 395, 130

Pei, Y. C., Fall, S. M., \& Hauser, M. G. 1999, ApJ, 522, 604

Perley, D. A., Cenko, S. B., Bloom, J. S., et al. 2009, AJ, 138, 1690

Pipino, A., Fan, X. L., Matteucci, F., et al. 2011, A\&A, 525, A61

Prochaska, J. X., Sheffer, Y., Perley, D. A., et al. 2009, ApJ, 691, L27

Savaglio, S., Glazebrook, K., \& Le Borgne, D. 2009, ApJ, 691, 182

Savaglio, S., Rau, A., Greiner, J., et al. 2012, MNRAS, 420, 627

Schady, P., Savaglio, S., Krühler, T., Greiner, J., \& Rau, A. 2011, A\&A, 525, A113

Schady, P., Dwelly, T., Page, M. J., et al. 2012, A\&A, 537, A15

Schneider, R., Omukai, K., Bianchi, S., \& Valiante, R. 2012, MNRAS, 419, 1566

Shin, M.-S., Berger, E., Penprase, B. E., et al. 2006, unpublished [arXiv:astro-ph/0608327]

Smith, M. W. L., Eales, S. A., Gomez, H. L., et al. 2012, ApJ, 756, 40

Starling, R. L. C., Wijers, R. A. M. J., Wiersema, K., et al. 2007, ApJ, 661, 787

Tanvir, N. R., Fox, D. B., Levan, A. J., et al. 2009, Nature, 461, 1254

Thöne, C. C., Kann, D. A., Jóhannesson, G., et al. 2010, A\&A, 523, A70

Thöne, C. C., Wiersema, K., Ledoux, C., et al. 2008, A\&A, 489, 37

Thöne, C. C., Fynbo, J. P. U., Goldoni, P., et al. 2013, MNRAS, 428, 3590

Toft, S., Hjorth, J., \& Burud, I. 2000, A\&A, 357, 115

Valiante, R., Schneider, R., Bianchi, S., \& Andersen, A. C. 2009, MNRAS, 397, 1661

Vladilo, G., Centurión, M., Levshakov, S. A., et al. 2006, A\&A, 454, 151

Vreeswijk, P. M., Ellison, S. L., Ledoux, C., et al. 2004, A\&A, 419, 927

Wang, J.-G., Zhou, H.-Y., Ge, J., et al. 2012, ApJ, 760, 42

Watson, D. 2011, A\&A, 533, A16

Watson, D., Fynbo, J. P. U., Ledoux, C., et al. 2006, ApJ, 652, 1011

Watson, D., Hjorth, J., Fynbo, J. P. U., et al. 2007, ApJ, 660, L101

Watson, D., Zafar, T., Andersen, A. C., et al. 2013, ApJ, 768, 23

Weingartner, J. C., \& Draine, B. T. 2001, ApJ, 548, 296

Zafar, T., Watson, D. J., Malesani, D., et al. 2010, A\&A, 515, A94

Zafar, T., Watson, D., Fynbo, J. P. U., et al. 2011a, A\&A, 532, A143

Zafar, T., Watson, D. J., Tanvir, N. R., et al. 2011b, ApJ, 735, 2

Zafar, T., Watson, D., Elíasdóttir, Á., et al. 2012, ApJ, 753, 82

Zhukovska, S., \& Gail, H.-P. 2009, in Cosmic Dust - Near and Far, eds. T. Henning, E. Grün, \& J. Steinacker, ASP Conf. Ser., 414, 199 